\title{
Destabilization of a flow focused suspension of magnetotactic bacteria
}

\author{
Nicolas Waisbord,${ }^{1}$ Christopher T. Lefèvre, ${ }^{2}$ Lydéric Bocquet, ${ }^{1, *}$ \\ Christophe Ybert, ${ }^{1}$ and Cécile Cottin-Bizonne ${ }^{1, \dagger}$ \\ ${ }^{1}$ Univ Lyon, Université Claude Bernard Lyon 1, CNRS, Institut Lumière Matière, F-69622, Lyon, France \\ ${ }^{2}$ Institut de Biosciences et Biotechnologies, UMR No. 7265, CEA Cadarache, CNRS, \\ Aix-Marseille Université, 13108 Saint Paul lez Durance, France
}

(Received 3 February 2016; published 21 September 2016)

\begin{abstract}
Active matter is a new class of intrinsically out-of equilibrium material with intriguing properties. The recent upsurge of studies in this field has mostly focused on the spontaneous behavior of these systems. Yet, many systems evolve under external constraints and driving forces, being subjected to both flow and various taxis. We present a new experimental system based on the directional control of magnetotactic bacteria which enables quantitative investigations to complement the challenging theoretical description of such systems. We explore the behavior of self-propelled magnetotactic bacteria as a particularly rich and versatile class of driven matter, whose behavior can be studied under a range of hydrodynamic and magnetic field stimuli. In particular we demonstrate that the competition between cell orientation toward a magnetic field and hydrodynamic flow lead not only to jetting, but to a new pearling instability. This model system illustrates new structuring capabilities of driven active matter.
\end{abstract}

DOI: 10.1103/PhysRevFluids.1.053203

\section{INTRODUCTION}

Active matter has been an emerging and rapidly growing field in the past decade. This research has led to the development of many numerical and theoretical investigations, complemented by (significantly fewer) experimental characterizations [1]. Studies on active particles have mostly focused on the spontaneous behavior of individual particles or on assemblages of active particles with the emergence of clusters or phase separation. Active matter is sometimes defined by the random unbiased motion of such particles [2]. Although bearing much significance, e.g., from a fundamental statistical physics point of view, many relevant practical situations actually involve additional external constraints. This encompasses the ubiquitous presence of surrounding liquid flows with essential consequences spanning from biological systems such as sperm cell motility [3] to the design of artificial microrobots in the vein of Feynman's dream for minimally invasive medicine [4]. In this situation, trajectories of spherical swimmers in Poiseuille flow, for instance, have been identified theoretically (i.e., swinging and tumbling) [5]. In addition, the possibility of bacterial localization mechanisms in flows, due to the so-called shape-induced rheotaxis, has been demonstrated $[6,7]$.

Beyond their interaction with the surrounding hydrodynamic environment, microswimmers can bias their motion in response to external stimuli through either passive (e.g., gravitational or magnetic fields) or active (e.g., toward chemical and optical fields) mechanisms. The ubiquitous ability of living active matter to bias its motion is of key importance in observed collective motions [8-10]. The understanding and modeling of active system behavior thus critically relies upon a model experimental system. The present work introduces magnetotactic bacteria as a promising model system of driven active matter that offers unprecedented capabilities for physical and quantitative investigations. This class of bacteria synthesizes nanomagnets in their cell membrane, providing

\footnotetext{
*Present address: Laboratoire de Physique Statistique, Ecole Normale Supérieure, UMR CNRS 8550, PSL Research University, 75005 Paris Cedex 05, France.

${ }^{\dagger}$ Cecile.Cottin-Bizonne@univ-lyon1.fr
} 
(a)

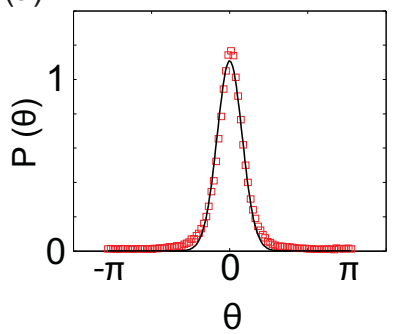

(b)

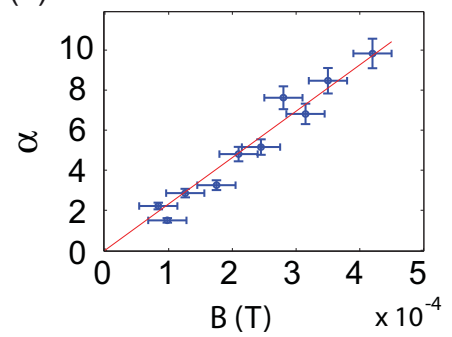

FIG. 1. Bacteria orientation. (a) Experimental statistics of orientations for a magnetic field of $0.42 \mathrm{mT}$ adjusted with $P(\theta) \propto e^{\alpha \cos (\theta)}$ as proposed by the Langevin model (see the text for details). (b) Evolution of $\alpha$ with the magnetic field; the red line is the linear adjustment $\alpha=B / B_{c}$ with a slope $1 / B_{c}=2.3 \pm 0.1 \times 10^{4} \mathrm{~T}^{-1}$.

a permanent magnet of the order of $M=1 \times 10^{-16} \mathrm{~A} \mathrm{~m}^{2}$ [11,12]. These magnetosomes enable them to be very easily remotely controlled by a magnetic field, which orients the motion of the bacteria by a magnetic torque [13]. The motion of the driven magnetotactic bacteria Magnetococcus marinus strain MC-1 is experimentally studied here in the presence of a steady Poiseuille flow. Those bacteria have been shown to be nearly spherical with a radius around $1 \mu \mathrm{m}$ [14]. At small flow velocities upstream swimming cells exhibit a focusing into a magnetotactic jet, reminiscent of observation in gravitactic or phototactic systems $[15,16]$, gathering both of the advantages of these two taxis: the physical simplicity of the action on the orientation of the first one and the easily and continuously tunable aspect of the second. Remarkably, this experimental biosystem is shown to quantitatively obey a simple physical description of Fokker-Planck type. We present a quantitative test of this widespread approach of active driven matter under flow. Increasing the flow velocities, we also demonstrate a type of instability leading to the development of recirculating swarms of magnetotactic bacteria. This sheds light on the structuring capabilities of driven active matter with possible outcomes in the many promising microswimmers able to orient with the homogeneous magnetic field $[17,18]$.

\section{ORIENTATION IN A MAGNETIC FIELD}

As a first step towards a quantitative description of the external driving, we characterize the ability of MC-1 bacteria to be directed by a magnetic field. MC-1 bacteria are placed in a microfluidic chamber in a still standing fluid. The bacteria display a smooth swimming motion modified by the rotational diffusion. We have studied their response to a uniform magnetic field B. Qualitatively, randomly oriented bacteria with an individual swimming velocity of $V_{\text {swim }}=100 \pm 10 \mu \mathrm{m} / \mathrm{s}$ get oriented by $\mathbf{B}$ and swim on average along the magnetic field lines [Fig. 1(a)]. More quantitatively, for a given magnetic field, the statistics of bacterial orientation show a distribution centered along the field direction, with a width associated with a finite orientational noise [Fig. 1(a)]. This experiment has long been considered as a way to estimate the magnetic momentum of bacteria, assuming that only the thermal bath contributes to orientational noise $[19,20]$. However, a recent study suggests that other sources can be involved in the cell disorientation phenomena [21].

The random torque $\zeta(t)$ responsible for random reorientations encompasses not only thermal fluctuations, but also tumbling and swimming noise, which we assume can be summed up in a total contribution that remains uncorrelated in time [7]:

$$
\left\langle\zeta(t) \zeta\left(t^{\prime}\right)\right\rangle=\xi^{2} \delta\left(t-t^{\prime}\right), \quad\langle\zeta(t)\rangle=0
$$

with $\xi$ the variance of the random torque. This problem remains analogous to the classical Langevin treatment with the orientation of the bacteria set by the equilibrium between (i) the magnetic torque $-M B \sin \theta$ (with $\mathbf{M}$ the magnetic momentum of the bacterium and $\theta$ the angle between the bacterium 
axis and B), (ii) the viscous torque $-\dot{\theta} / \mu_{R}$ [with $\mu_{R}=\left(8 \pi \eta r^{3}\right)^{-1}$ the mobility, $r$ the hydrodynamic radius, and $\eta$ the viscosity of the liquid], and (iii) the random torque $\zeta(t)$. This predicts a probability orientation matching Langevin paramagnetism, with an a priori unknown noise amplitude (see [22])

$$
P(\theta) \propto \exp \left(\frac{2 M B}{\xi^{2} \mu_{R}} \cos \theta\right) \propto \exp \left(\frac{B}{B_{c}} \cos \theta\right),
$$

with $B_{c}$ a characteristic magnetic field scale defined by

$$
B_{c}=\frac{\xi^{2} \mu_{R}}{2 M} .
$$

As can be seen in Fig. 1(a), the orientation distribution of bacteria is very well described by the Langevin prediction $\exp (\alpha \cos \theta)$ [Eq. (2)]. Plotting $\alpha$ versus $B$ in Fig. 1(b), we demonstrate the validity of the linear dependence in $B$ expected from Eq. (2) with $\alpha \equiv B / B_{c}$ and obtain $B_{c}=$ $43 \pm 2 \mu \mathrm{T}$. Despite the fact that there might be multiple rotational diffusion sources in these living microswimmers, the balance with magnetic torque is hence fully summed up in this characteristic magnetic field that we can measure for magnetotactic bacteria and that we might be able to choose for artificial magnetic microswimmers.

\section{FLOW FOCUSED SUSPENSION}

We are now in a position to quantitatively investigate the interplay between magnetically driven active matter and an external flow field. This is done using a straight microfluidic channel of cross section $2 w \times 2 w=50 \times 50 \mu \mathrm{m}^{2}$, where the response of the magnetotactic microswimmers to steady Poiseuille flow is explored. We focus in the following on the situation where the magnetic microswimmers are oriented against the fluid flow and along the channel axis. While the flowing suspension remains fully homogeneously distributed over the channel width in the absence of an imposed magnetic field, we observe that it rapidly concentrates in a narrower jet at the center of the channel upon turning the field on [Fig. 3(a)]. Bacteria accumulate at the channel walls when swimmers are magnetically oriented in the flow direction (not shown).

This behavior is reminiscent of similar focusing phenomena reported for gyrotactic or phototactic motile algal cells $[15,16]$. However, so far no quantitative experimental testing of gyrotaxis has been carried out [23]. Beyond the demonstration of this phenomenon for magnetotactic bacteria, a key aspect of this model system is that the characterization of the focusing mechanism can be made fully quantitative and can be remotely and continuously controlled by the external field. Starting from a homogeneous flowing suspension, the latter focuses within $0.2 \mathrm{~s}$ (of order $w / V_{\text {swim }}$ ) in a stationary magnetotactic bacteria jet. By varying the magnitude of $\mathbf{B}$, it is possible to change the efficiency of the trapping: The width of the focused suspension decreases for increasing magnetic driving.

Neglecting the out-of-plane component of the swimmers' velocity, we consider a bidimensional problem characterized by a Poiseuille flow with a velocity field $\mathbf{u}$ and a maximum velocity $V_{\text {flow }}$ in a channel of width $2 w$. As sketched in Fig. 2, the bacterium is now submitted to a hydrodynamic torque, a magnetic torque, and a rotational noise. For the sake of simplicity we assume in this model that the chain axis is aligned with the flagellar position.

The hydrodynamic torque acting on a bacterium is

$$
\boldsymbol{\Gamma}_{\text {hyd }}=\frac{(1 / 2) \boldsymbol{\nabla} \times \mathbf{u}-\boldsymbol{\Omega}}{\mu_{R}},
$$

where $\boldsymbol{\Omega}$ is the bacterium angular velocity. For small enough flow velocities, a stationary orientation of the bacterium $(\Omega=0)$ is possible at every location in the channel:

$$
\Gamma_{\text {hyd }}=-K \frac{x}{w},
$$

with $K=\frac{V_{\text {flow }}}{\mu_{R} w}$ and $x$ the distance from the channel center. 


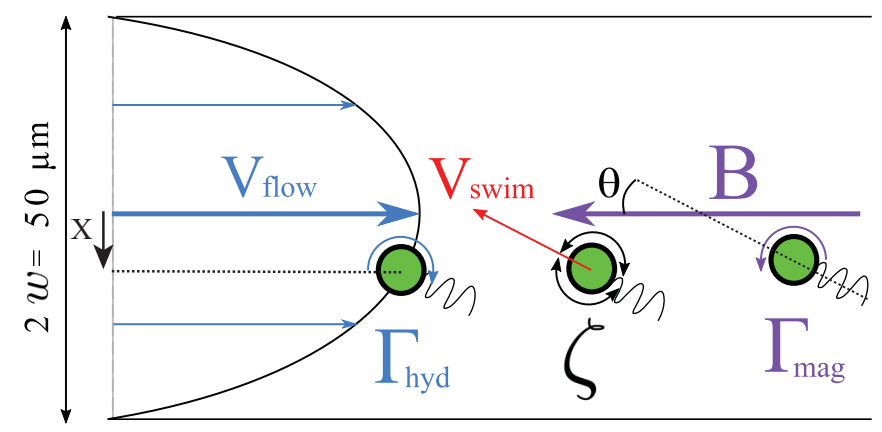

FIG. 2. Schematic representation of the magnetotactic bacteria in a Poiseuille flow, with a magnetic field oriented against the flow. The bacteria are submitted to a hydrodynamic torque $\boldsymbol{\Gamma}_{\text {hyd }}$, a magnetic torque $\boldsymbol{\Gamma}_{\text {mag }}$, and a random torque $\zeta$.

The magnetic torque is expressed as a function of the transverse speed $\dot{x}$ given that $\mathbf{V}_{\text {swim }}=$ $\dot{x} \mathbf{e}_{x}+\mathbf{V}_{\text {axial }}$, where $\mathbf{V}_{\text {axial }}$ is the axial speed:

$$
\boldsymbol{\Gamma}_{\mathrm{mag}}=\mathbf{M} \times \mathbf{B}=-M B \frac{\dot{x}}{V_{\mathrm{swim}}} .
$$

In this counterflow orientation for $\mathbf{B}$, the combined action of the magnetic field and the hydrodynamical shear drives the bacteria toward the center of the channel:

$$
0=M B \frac{\dot{x}}{V_{\text {swim }}}+K \frac{x}{w} .
$$

Generalizing the torque balance to account for the rotational noise $\zeta$ that is responsible for the finite extension of the focused jet, the bacterium radial trajectories obey the overdamped Langevin equation

$$
0=M B \frac{\dot{x}}{V_{\text {swim }}}+K \frac{x}{w}+\zeta
$$

Note that the low-velocity conditions for stationary orientation amount to $K<M B$ : The magnetic torque exceeds the maximum hydrodynamic torque arising at the channel walls.

This Ornstein-Uhlenbeck process is formally equivalent to the one of a damped Brownian particle in a harmonic potential, with the hydrodynamical torque $K$ playing the role of a harmonic potential. Solving the resulting Fokker-Planck equation leads to a Gaussian transverse distribution of the suspension for the steady-state bacterium density (see [22]):

$$
d(x) \propto e^{-x^{2} / 2 \ell^{2}}
$$

with a width $\ell$ defined by

$$
\ell=w \sqrt{\frac{V_{\text {swim }} B_{c}}{V_{\text {flow }} B}} .
$$

In line with the experimental observation, the model predicts that the focusing increases with increasing magnetic field. The same is expected when increasing the velocity, as it orients the swimmers farther apart from the axial field direction, towards the channel center. More quantitatively, the predicted Gaussian distribution is very well obeyed by the experimental bacterium density profiles as shown in Fig. 3(b). Investigating the profile width $\ell$ as a function of $B$, we moreover evidence the $1 / \sqrt{B}$ evolution [Fig. 3(c)]. Note that focusing widths at low magnetic fields seem to level off and to depart from the high field behavior. This departure is expected to occur due to a breakdown of the stationary orientation assumption throughout the whole channel. This requires $B>1.2 \pm$ $0.3 \mathrm{mT}$ [22], which is in very good agreement with the observed departure [blue circles in Fig. 3(c)]. 
(a)

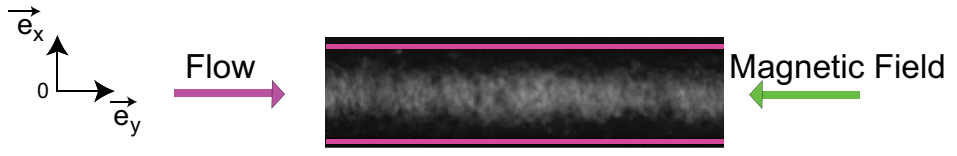

(b)

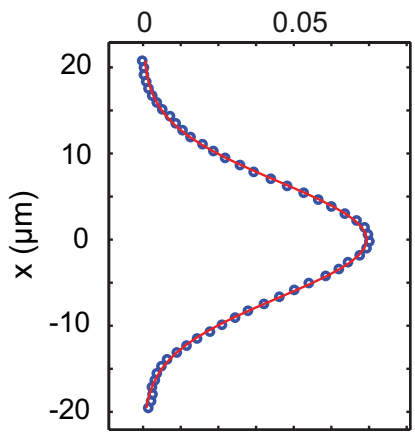

(c)

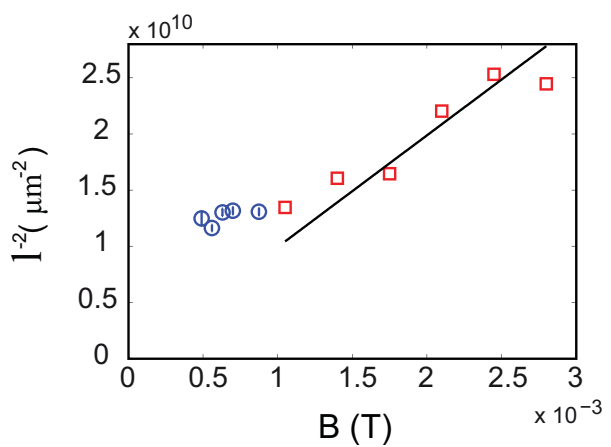

FIG. 3. Flow focused state when bacteria are driven upstream, with the swimming direction imposed by the magnetic field antiparallel to the fluid flow. (a) Picture of the system in a stationary state when bacteria are flushed with a central fluid velocity $V_{\text {flow }}=90 \pm 20 \mu \mathrm{m} / \mathrm{s}$ in a $50-\mu \mathrm{m}$-wide channel and a magnetic field ( $B=2.8 \mathrm{mT}$ ) tends to orient them in the opposite direction. (b) Corresponding transverse intensity profile, adjusted with a Gaussian fit. (c) Evolution of the width of the Gaussian profile with the magnetic field for $V_{\text {flow }}=90 \pm 20 \mu \mathrm{m} / \mathrm{s}$. The red squares are adjusted by a linear fit $\ell^{-2}=1.0 \times 10^{13} \mathrm{~m}^{-2} T^{-1} \times B$. The blue circles correspond to the regime where the stationary orientation assumption is no longer valid.

In addition to the focusing process, it is also possible to adjust the jet location within the channel by tilting the magnetic field direction by an angle $\beta$ with respect to the channel axis. This is shown in Fig. 4(a), where a suspension faces a central fluid velocity of half the typical swimming speed ( $V_{\text {flow }}=50 \mu \mathrm{m} / \mathrm{s}$ ), with a misalignment of an angle $\beta=8^{\circ}$. While still focused, the suspension jet is now shifted off center, towards the channel side. Overall, the Gaussian shape of the profile is maintained [Fig. 4(b)] with a peak offset $x_{\text {eq }}$ and a truncation due to the presence of the wall. Expanding on the previous theoretical description, the focusing location that corresponds to the expected maximum density is (see [22])

$$
x_{\text {eq }}=w \frac{M B}{K} \sin \beta .
$$

Figure 4(c) presents the evolution of the focusing position as a function of the misalignment $\beta$. The predicted linear dependence of $x_{\mathrm{eq}}$ in $\sin \beta$ is very well evidenced. This allows an experimental determination of $M B / K=2.1 \pm 0.2$. The consistency of this value can be checked by estimating the associated hydrodynamic radius of the bacteria:

$$
r=\left(\frac{K w}{8 \pi \eta V_{\text {flow }}}\right)^{1 / 3} .
$$

Considering a magnetic momentum $M=1 \pm 0.2 \times 10^{-16} \mathrm{~A} \mathrm{~m}^{2}[20,21], V_{\text {flow }}=50 \pm 10 \mu \mathrm{m} / \mathrm{s}$, $B=1.4 \mathrm{mT}$, and $w=25 \pm 5 \mu \mathrm{m}$, we obtain a hydrodynamic radius for bacteria $r=1.1 \pm 0.2 \mu \mathrm{m}$, which perfectly agrees with the size of the core bacteria, obtained with scanning electronic microscopy, around $1 \mu \mathrm{m}$ [14].

Overall, the magnetotactic bacteria with their magnetic driving of physical nature, matching the Langevin paramagnetism, provide a model system of driven active microsystems, whose focusing interaction with nonuniform flow can be fully quantitatively captured. Remarkably, this complex 
(a)

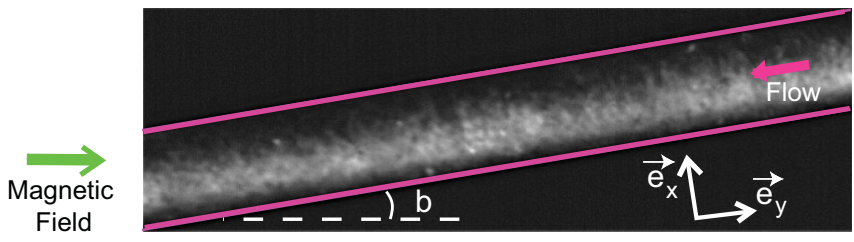

(b)

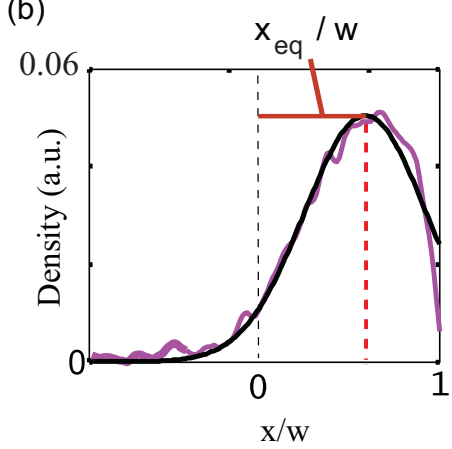

(c)

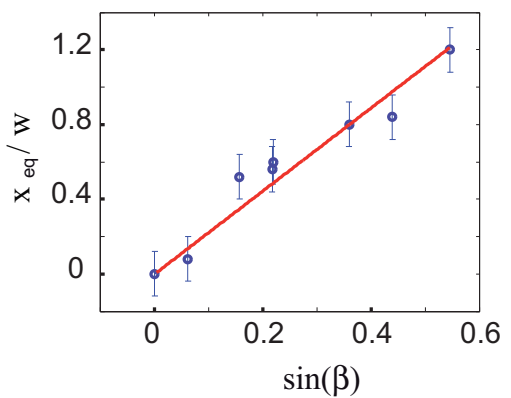

FIG. 4. Moving the trap position. (a) Sketch of the experiment, for $\beta=8^{\circ}, w=50 \mu \mathrm{m}$, and $V_{\text {flow }}=$ $50 \mu \mathrm{m} / \mathrm{s}$. (b) Experimental density profile adjusted with a shifted Gaussian fit. (c) Dependence of the trap position $x_{\text {eq }} / w$ with the tilting angle, for experiments with a magnetic field of $1.4 \mathrm{mT}$ and $V_{\text {flow }}=50 \mu \mathrm{m} / \mathrm{s}$. We adjust the position with $x_{\text {eq }} / w=A \sin \beta$ and obtain $A=2.1$.

biosystem is fully described by a simple Fokker-Planck equation. This constitutes a direct validation of such approaches initially proposed in the context of gyrotactic systems [23], which are used as a key element for the description of complex bioconvection patterns and dynamics in such systems.

Upstream swimming occurs naturally in pressure-driven flows of bacteria in the absence of any field [24], but an interesting aspect of externally driven systems is that they could serve as conceptual building blocks for remotely controllable microsurgeons or cargos that would be piloted through blood vessel networks [25]. In that perspective, shifting the focused microswimmers beam towards the channel side where the flow slows down could be a strategy for achieving upstream swimming against high-speed blood flow.

\section{JET PEARLING TRANSITION}

So far, we have explored the interaction of driven magnetotactic bacteria and surrounding Poiseuille flows at moderate flow velocities. In this regime, a stationary suspension focusing was obtained and increasing the magnetic field led to the concentration of the bacteria into a thinner jet. However, further increasing the external field or increasing the flow velocity induces a striking change of behavior. The originally stable beam of bacteria is destabilized into a pearling jet, which can yield to swarming droplets as shown in Fig. 5 (movies are available in Ref. [22]). Note that if we keep the same fluid flow but now lower the magnetic field, the focused suspension remains stable.

To carefully explore the $\left(V_{\text {flow }}, B\right)$ phase space, we fill the channel with magnetotactic bacteria and for different imposed $B$ we vary the fluid flow $V_{\text {flow }}$ oriented against the magnetic field. We then determine the conditions for which the focused jet remains stable or parts into suspension droplets. This yields a phase diagram presented in Fig. 5 showing two different dynamical regimes: a slow-flow low-field regime corresponding to the previously described stable focused jet and a fast-flow high-field regime for which the suspension parts into droplets.

A natural argument for active systems, intimately associated with the onset of collective motions, would be to relate the instability to the appearance of bacteria-bacteria interactions. Indeed, 


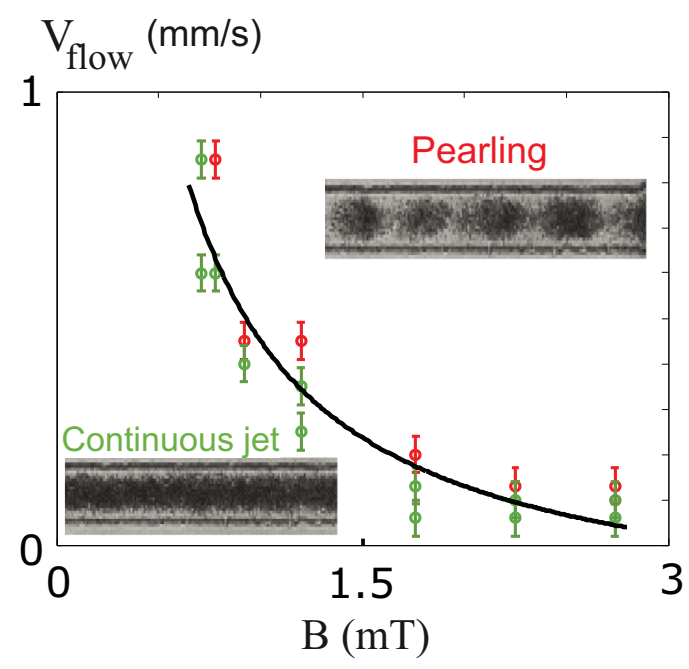

FIG. 5. Phase diagram of the instability for various values of $V_{\text {flow }}$ and $B$. Red circles are the unstable jets, green circles are the stable jets. The black line is the best adjustment with $V_{\text {flow }}=a / B+c$ with $a=0.630 \mathrm{~mm}$ $\mathrm{mT} / \mathrm{s}$ and $c=-0.19 \mathrm{~mm} / \mathrm{s}$. The density in bacteria in this experiment is of the order of $10^{7}$ bacteria $/ \mu \mathrm{L}$.

increasing the flow velocity or the magnetic field confines the bacteria in an increasingly smaller jet. Thus, the jet density goes as $\rho_{0}(w / \ell)^{2}$, with $\rho_{0}$ the initially homogeneous bacteria density and $\ell$ the jet radius as given by Eq. (10). Associating the transition with a threshold density, one would expect a simple relationship between $B$ and $V_{\text {flow }}, V_{\text {flow }}^{\text {th }} \propto 1 / B_{\text {th }}$, in fair agreement with the experimental threshold (Fig. 5). Considering $\rho_{0} \sim 10^{7}$ bacteria/ $\mu \mathrm{L}$, this would translate into a volume fraction of $\phi \sim 32 \%$ in the jet. This suggests that bacteria-bacteria interactions might play a role in the present pearling instability.

We take advantage of the unprecedented control offered by the magnetotactic bacteria to provide some additional experimental insight into the pearling transition characteristics. We quantified the breakdown of the jet homogeneity by increasing the magnetic field at a fixed velocity, set here to $V_{\text {flow }}=300 \pm 40 \mu \mathrm{m} / \mathrm{s}$. To characterize the modulation of the jet properties, we computed the mean axial density profile (over the field of view, typically $200 \mu \mathrm{m}) \hat{\rho}(y)=\langle\rho\rangle_{x}$ and defined the contrast parameter as

$$
C=\frac{\hat{\rho}_{\max }-\hat{\rho}_{\min }}{\hat{\rho}_{\max }+\hat{\rho}_{\min }},
$$

where $\hat{\rho}_{\max }\left(\hat{\rho}_{\min }\right)$ is the maximum (minimum) density along the channel. Starting from a low magnetic field, stable jet situation, the target field is imposed at time $t=0$ and we look at the subsequent evolution of the jet contrast over time, as shown in Fig. 6(a). For $B=0.35 \mathrm{mT}$ the contrast has a unique steady value over the duration of observation (black curve): There is a homogeneous and stationary focused beam of bacteria. The small drift observed is due to the small time evolution of the density in bacteria. At a higher magnetic field of $B=2.1 \mathrm{mT}$, for which we are above the pearling instability threshold, the contrast shows a very distinct time evolution. Starting from a steady value corresponding to the focused beam, the contrast gradually increases until it plateaus again at a higher contrast $(\Delta C)$ corresponding here to the flow of the bacteria droplets. Figure $6(\mathrm{~b})$ presents the measured contrast increase as a function of the applied field $B$ with fixed flow velocity. The transition from a stable flow focused state to a pearling jet is very clearly revealed at $B_{\text {th }}=0.83 \mathrm{mT}$, with $\Delta C$ departing from zero and further increasing as $B$ gradually moves above the threshold. Qualitatively, this looks like a supercritical Hopf bifurcation between a stable homogeneous jet and a varicose modulation. This suggests that the modulation amplitude might evolve like the square 
(a)
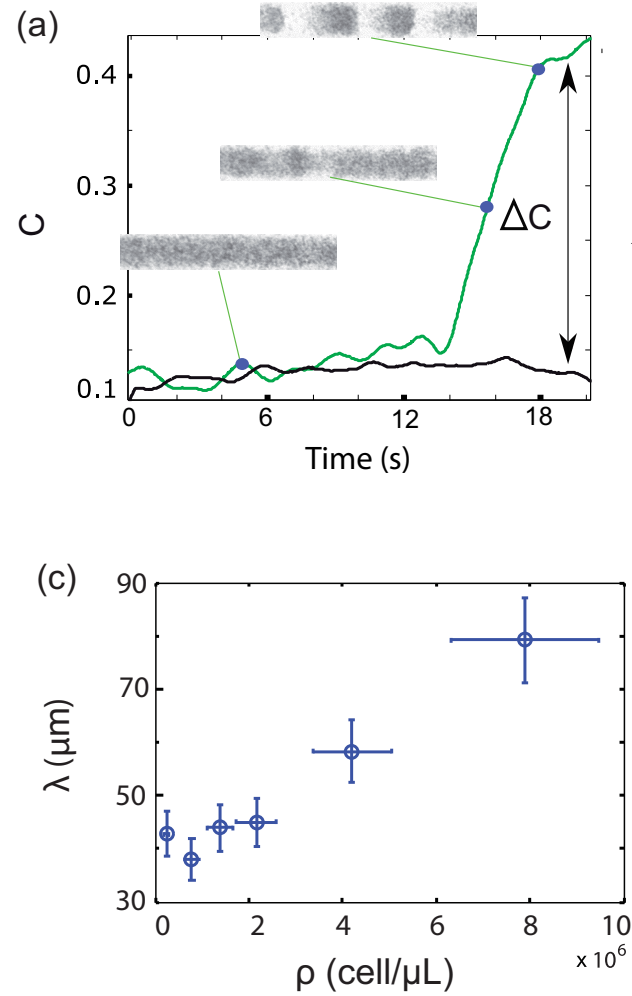

(e)

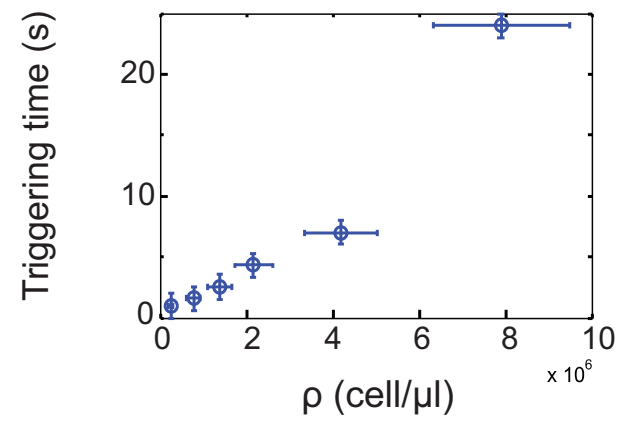

(b)
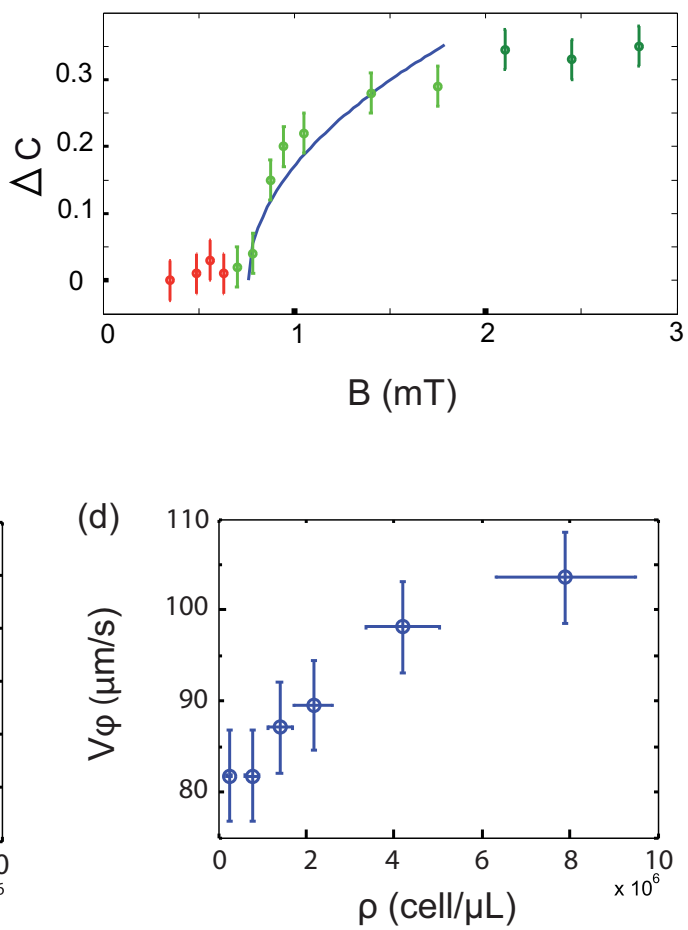

(f)

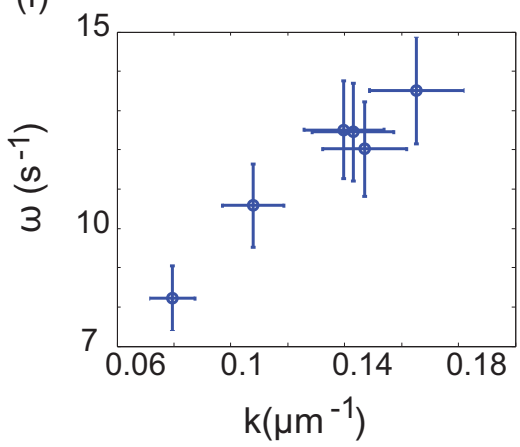

FIG. 6. Characterizations of the instability. (a) Evolution of the spatial contrast of the jet during flushing at $V_{\text {flow }}=300 \pm 40 \mu \mathrm{m} / \mathrm{s}$, in green at $2.1 \mathrm{mT}$, in black at $0.3 \mathrm{mT}$. The blue dots correspond to the values of the parameters for which the pictures are taken. (b) Evolution of the contrast burst $\Delta C$ with B for a central fluid velocity of $V_{\text {flow }}=300 \pm 40 \mu \mathrm{m} / \mathrm{s}$, and the adjustment by $\left(B-B_{\text {th }}\right)^{1 / 2}$ with $B_{\text {th }}=0.83 \mathrm{mT}$. In (c)-(f) $B=2.8 \mathrm{mT}$ and $V_{\text {flow }}=180 \mu \mathrm{m} / \mathrm{s}$. (c) Evolution of the wavelength with the initial density. A net increase of the wavelength appears as the density increases. (d) Evolution of the phase speed of the traveling wave in the laboratory frame as a function of the initial density. (e) Evolution of the triggering time of the instability with the initial density. (f) Dispersion relation $\omega$ as a function of the wave number $k$.

root of the distance to threshold, in fair agreement with the experimental behavior as shown by the adjustment of data by

$$
\Delta C \propto\left(B-B_{\mathrm{th}}\right)^{1 / 2} .
$$




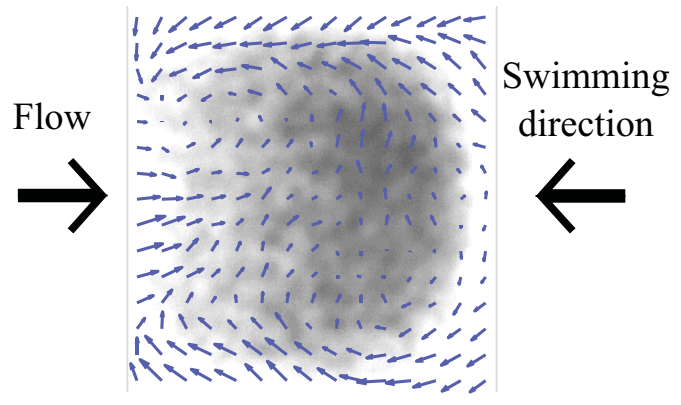

FIG. 7. Tracked swarming droplet. With the superposed bacteria velocities, one can easily distinguish a recirculation inside the droplet.

We performed additional measurements as a function of the bacterial density. We have used a bacteria suspension of slowly varying density (see [22] for experimental details), from which two different data have been extracted.

First, while the focusing time for creating the bacteria jet is always rapid (less than $0.3 \mathrm{~s}$ ), the triggering time for the suspension to destabilize can be greater than $20 \mathrm{~s}$. More quantitatively, we observe [Fig. 6(e)] that the triggering time increases with bacteria density for fixed values of magnetic field and Poiseuille flow (here $B=2.8 \mathrm{mT}$ and $V_{\text {flow }}=180 \mu \mathrm{m} / \mathrm{s}$ ). In contrast, we did not observe a change of triggering time for fixed density but we did for variable magnetic field amplitudes. Second, we have also measured the characteristic length scale of the instability pattern as a function of the density. As shown in Fig. 6(c), this wavelength increases with the initial density.

Measuring the phase speed of the jet modulation [Fig. 6(d)], we are also able to obtain a portion of the dispersion relation for the instability [Fig. 6(f)]. The phase speed appears quite different from the Poiseuille central velocity (here $V_{\text {flow }}=180 \mu \mathrm{m} / \mathrm{s}$ ) and much more compatible with an almost fixed modulation in a frame traveling at $V_{\text {flow }}-V_{\text {swim }}$.

As a final experimental characterization of the instability, we report in Fig. 7 the relative velocities of bacterial swarm once the pearl has fully segregated. We observe an inner circulation composed of two counterrotating bacterial vortices, showing the complexity of the coupling between the flow and the bacteria motion, in good agreement with the dependence of the phase speed with the density exhibited in Fig. 6(d). This suggests a feedback of the active suspension on the fluid velocity. At very high density, one can expect that steric interactions will reduce this recirculation and hence slow down the destabilization process. Quite remarkably the density of the interacting objects increases the size of the final pattern obtained, but the dynamics to obtain this pattern is slowed down by the density.

\section{DISCUSSION: CHALLENGING THEORIES}

A theoretical description of this instability requires identifying the relevant ingredients involved in this complex system: a driven active system under flow. Of course liquid jet instabilities are known to occur also in purely passive (i.e., equilibrium) systems, for instance, due to Kelvin-Helmholtz or Rayleigh-Plateau systems [26]. However, thinking along these lines would require defining for the effective active fluid physical properties such as viscosity or surface tensions. If some viscosities of active systems have been characterized [27-30], there has been, so far, no studies on the viscosity of suspensions of magnetotactic bacteria. As for the idea of generalizing the surface tension in active systems, it has only emerged very recently [31]. Indeed, the mere notion of active pressure was recently introduced [32-34] and here, with field oriented swimmers, active pressure anisotropy and active surface tensions might be expected. However, the practical meaning and usefulness of these different variables are still a matter of extensive theoretical research [35] that precludes its current use for drawing generalization from passive systems instabilities. One should also notice that the true nature of the Poiseuille flow might also be altered at very high bacterial concentration, by the 
flow field generated by the self-propulsion of the bacteria and by the torque exerted by the magnetic field on the fluid via the bacteria.

Nevertheless, active matter has primarily attracted attention because of the spontaneous appearance of collective motions associated with spatiotemporal coherent structures, for instance, in various biological micro-organisms [36]. Thus instabilities and pattern formation specific to active systems have been the subject of intense research $[8,9,40]$. Of particular interest here are gravitactic and phototactic systems, which share with magnetotaxis the ability of being remotely oriented by an external field.

Recent experiments on phototactic swimmers [16] indeed reported the focusing regime, yet without noticing jet instabilities. A subsequent numerical paper considering puller phototactic swimmers submitted to a Poiseuille flow showed a pearling instability [37]. However, despite that this reported instability was assigned to hydrodynamic interactions between swimmers, it seems to depart from the present one. Indeed, no instability threshold, meaning no stable focused jet, is found numerically. Additionally, the self-focusing time is found to be comparable to the subsequent time for jet breakup into swimmer pearls, while these time scales are quite far apart in the present study. Moreover, besides the phenomenology it only gives a qualitative picture for the instability based on the puller-puller interactions.

Concerning gravitactic bio-organisms, they have long been known to exhibit gyrotaxis [15], where gravity plays a role similar to the present magnetic field and bottom-heavy cells orient by a balance between gravitational and shear torques. There, self-focused jets have been observed that might give rise to blip instability as reported experimentally $[15,38]$. Here again, the instabilities do not seem to have the same behaviors and properties. While a velocity threshold is now also observed, gyrotactic swimmers display a restabilization of the jet at high flow velocities, in strong contrast to our findings. The phase speed of the jet modulation in gyrotactic swimmers was also reported to be close to the central velocity [15,38], which is not what we observe with our system. Qualitatively this magnetotactic instability looks like a varicose mode with definite characteristic length scales evolving toward isolated swarms, while gyrotactic algae are shown to yield very distant concentrated drops of swimmers connected with a thin filament or a radius transition between coexisting jets [38].

On the theoretical side, much has been done on gyrotactic instabilities $[8,9,39,40]$ owing to their relevance for bioconvection, organic matter plumes, etc. [15,41,42]. However, the instability reported herein for magnetotactic bacteria lacks a key ingredient usually involved in the previously mentioned phenomenology. Namely, gyrotactic systems do incorporate a gravitational body force that couples the flow field with the local micro-organisms' density, due to the local density mismatch. For magnetotactic bacteria, it is important to stress that no such equivalent exists as no force is exerted on the bacteria by the field. In recent studies of jet stability of gyrotactic swimmers [43], this body-force term is found to be of major importance for the explored instabilities

Therefore, direct comparison with our system is difficult. Moreover, discussion is made in the absence of swimmer interactions (the swimming stress contribution [43-45] has been neglected), while a simple analysis of the instability thresholds suggests here that it plays a significant role.

\section{CONCLUSION}

To summarize, we have unveiled rich behaviors of driven active matter subjected to an external field. We have studied a model of an active system comprised of magnetotactic bacteria whose motion can be tuned and oriented by an external magnetic field. When facing a Poiseuille flow, at moderate velocity and low magnetic field, the suspension is focused in the center of the channel, reminiscent of similar behaviors observed for phototactic or gravitactic systems. We propose a simple stochastic model that allows quantitative prediction of the strength and the position of the trap as a function of the direction and the magnitude of the external driving field. Providing a quantitative description of these systems is a key element for building models to understand the complex pattern formation of microswimmers colonies. For higher values of the magnetic field or the velocity of the imposed flow, we observe a transition towards a state where the bacterial suspension destabilizes 
in swarming droplets. This instability illustrates structuring capabilities of driven active matter and challenges actual theoretical frameworks of active matter, suggesting further horizons to explore in this extremely rich domain.

\section{ACKNOWLEDGMENTS}

We thank Siddardha Koneti for his work on the instability and Veroniquie Utzinger, Guy Condemine, and Nicole Cotte-Pattat for their help with the bacterial cultures. We thank INL for access to their clean room facilities and AXA research fund for its financial support.

[1] M. Cates, Diffusive transport without detailed balance in motile bacteria: Does microbiology need statistical physics? Rep. Prog. Phys. 75, 042601 (2012).

[2] S. Ramaswamy, The mechanics and statistics of active matter, Annu. Rev. Condens. Matter Phys. 1, 323 (2010).

[3] J. Riffell and R. Zimmer, Sex and flow: The consequences of fluid shear for sperm-egg interactions, J. Exp. Biol. 210, 3644 (2007).

[4] R. P. Feynman, Plenty of room at the bottom, Annual Meeting of the American Physical Society at California Institute of Technology, 1959, available at http://www.zyvex.com/nanotech/feynman.html

[5] A. Zotl and H. Stark, Nonlinear Dynamics of a Microswimmer in Poiseuille Flow, Phys. Rev. Lett. 108, 218104 (2012).

[6] Marcos, H. C. Fu, T. R. Powers, and R. Stocker, Separation of Microscale Chiral Objects by Shear Flow, Phys. Rev. Lett. 102, 158103 (2009).

[7] R. Rusconi, J. S. Guasto, and R. Stocker, Bacterial transport suppressed by fluid shear, Nat. Phys. 10, 212 (2014).

[8] B. Ezhilan, M. J. Shelley, and D. Saintillan, Instabilities and nonlinear dynamics of concentrated active suspensions, Phys. Fluids 25, 070607 (2013).

[9] D. L. Koch and G. Subramanian, Collective hydrodynamics of swimming microorganisms: Living fluids, Annu. Rev. Fluid Mech. 43, 637 (2011).

[10] T. J. Pedley and J. O. Kessler, Hydrodynamic phenomena in suspensions of swimming microorganims, Annu. Rev. Fluid Mech. 24, 313 (1992).

[11] D. A. Bazylinski and R. Frankel, Magnetosome formation in prokaryotes, Nat. Rev. Microbiol. 2, 217 (2004).

[12] F. C. Meldrum, B. R. Heywood, S. Mann, R. B. Frankel, and D. A. Bazylinsly, Electron microscopy study of magnetosomes in a cultured coccoid magnetotactic bacterium, Proc. R. Soc. London B 251, 231 (1993).

[13] S. Martel, M. Mohammadi, O. Felfoul, L. Zhao, and P. Pouponneau, Flagellated magnetotactic bacteria as controlled MRI-trackable propulsion and steering systems for medical nanorobots operating in the human microvasculature, Int. J. Robot. Res. 28, 571 (2009).

[14] D. A. Bazylinski, T. J. Williams, C. T. Lefevre, R. J. Berg, C. L. Zhang, S. S. Bowser, A. J. Dean, and T. J. Beveridge, Magnetococcus marinus gen. nov., sp. nov., a marine, magnetotactic bacterium that represents a novel lineage at the base of the alphaproteobacteria, Int. J. Syst. Evol. Microbiol. 63, 801 (2013).

[15] J. O. Kessler, Hydrodynamic focusing of motile algal cells, Nature (London) 313, 218 (1985).

[16] X. Garcia, S. Rafaï, and P. Peyla, Light Control of the Flow of Phototactic Microswimmer Suspensions, Phys. Rev. Lett. 110, 138106 (2013).

[17] A. Ghosh and P. Fischer, Controlled Propulsion of Artificial Magnetic Nanostructured Propellers, Nano Lett. 9, 2243 (2009).

[18] W. Gao, X. Feng, A. Pei, C. R. Kane, R. Tam, C. Hennessy, and J. Wang, Bioinspired helical microswimmers based on vascular plants, Nano Lett. 14, 305 (2014).

[19] R. P. Blakemore, Magnetotactic bacteria, Annu. Rev. Microbiol. 36, 217 (1982).

[20] R. Nadkarni, S. Barkley, and C. Fradin, A comparison of methods to measure the magnetic moment of magnetotactic bacteria through analysis of their trajectories in external magnetic fields, PLoS ONE 8, e82064 (2013). 
[21] X. Zhu, X. Ge, N. Li, L. F. Wu, C. Luo, Q. Ouyang, and G. Chen, Angle sensing in magnetotaxis of Magnetospirillum magneticum AMB-1, Integrat. Biol. 6, 706 (2014).

[22] See Supplemental Material at http://link.aps.org/supplemental/10.1103/PhysRevFluids.1.053203 for experimental and theoretical details and a typical movie of the instability.

[23] T. J. Pedley, Gyrotaxis in uniform vorticity, J. Fluid Mech. 762, R6 (2015).

[24] B. Ezhilan and D. Saintillan, Transport of a dilute active suspension in pressure-driven flow, J. Fluid Mech. 777, 482 (2015).

[25] O. Felfoul, M. Mohammadi, S. Taherkhani, D. de Lanauze, Y. Z. Xu, D. Loghin, S. Essa, S. Jancik, D. Houle, M. Lafleur, L. Gaboury, M. Tabrizian, N. Kaou, M. Atkin, T. Vuong, G. Batist, N. Beauchemin, D. Radzioch, and S. Martel, Magneto-aerotactic bacteria deliver drug-containing nanoliposomes to tumour hypoxic regions, Nat. Nanotechnol. (2016), doi: 10.1038/nnano.2016.137.

[26] J. Eggers and E. Villermaux, Physics of liquid jets, Rep. Prog. Phys. 71, 036601 (2008).

[27] D. Saintillan, The dilute rheology of swimming suspensions: A simple kinetic model, Exp. Mech. 50, 1275 (2009).

[28] A. Sokolov and I. Aranson, Reduction of Viscosity in Suspension of Swimming Bacteria, Phys. Rev. Lett. 103, 148101 (2009).

[29] S. Rafaï, L. Jibuti, and P. Peyla, Effective Viscosity of Microswimmer Suspension, Phys. Rev. Lett. 104, $098102(2010)$.

[30] J. Gachelin, G. Mino, H. Berther, A. Lindner, A. Rousselet, and E. Clément, Non-Newtonian Viscosity of Escherichia Coli Suspensions, Phys. Rev. Lett. 110, 268103 (2013).

[31] J. Bialké, J. T. Siebert, H. Löwen, and T. Speck, Negative Interfacial Tension in Phase-Separated Active Brownian Particles, Phys. Rev. Lett. 115, 098301 (2015).

[32] S. C. Takatori, W. Yan, and J. F. Brady, Swim Pressure: Stress Generation in Active Matter, Phys. Rev. Lett. 113, 028103 (2014).

[33] X. Yang, M. L. Manning, and M. C. Marchetti, Aggregation and segregation of confined active particles, Soft Matter 10, 6477 (2014).

[34] A. P. Solon, J. Stenhammar, R. Wittkowski, M. Kardar, Y. Kafri, M. E. Cates, and J. Tailleur, Pressure and Phase Equilibria in Interacting Active Brownian Spheres, Phys. Rev. Lett. 114, 198301 (2015).

[35] A. P. Solon, Y. Fily, A. Baskaran, Mickael E. Cates, Y. Kafri, M. Kardar, and J. Tailleur, Pressure is not a state function for generic active fluids, Nat. Phys. 11, 673 (2015).

[36] T. Ishikawa, Suspension biomechanics of swimming microbes, J. R. Soc. Interface 6, 815 (2009).

[37] L. Jibuti, L. Qi, C. Misbah, W. Zimmermann, S. Rafaï, and P. Peyla, Self-focusing and jet instability of a microswimmer suspension, Phys. Rev. E 90, 063019 (2014).

[38] P. Denissenko and S. Lukaschuk, Velocity profiles and discontinuities propagation in a pipe flow of suspension of motile microorganisms, Phys. Lett. A 362, 298 (2007).

[39] D. Saintillan, Swimming in shear, J. Fluid Mech. 744, 1 (2014).

[40] N. A. Hill and T. J. Pedley, Bioconvection, Fluid Dyn. Res. 37, 1 (2005).

[41] J. O. Kessler, Individual and collective fluid dynamics of swimming cells, J. Fluid Mech. 173, 191 (1986).

[42] J. R. Platt, Bioconvection patterns in cultures of free-swimming organisms, Science 133, 1766 (1986).

[43] Y. Hwang and T. J. Pedley, Stability of downflowing gyrotactic microorganism suspensions in a twodimensional vertical channel, J. Fluid Mech. 749, 750 (2014).

[44] D. Saintillan and M. J. Shelley, Instabilities, pattern formation, and mixing in active suspensions, Phys. Fluids 20, 123304 (2008).

[45] R. A. Simha and S. Ramaswamy, Hydrodynamic Fluctuations and Instabilities in Ordered Suspensions of Self-Propelled Particles, Phys. Rev. Lett. 89, 058101 (2002). 